\title{
Bidirectional Dependency of Developmental and Social Difficulties in Hearing Impaired Children on the Mother's State Anxiety
}

\author{
Agnieszka Pilarska DD $^{1,2} \cdot$ Alicja Sekula ${ }^{1,2}$
}

Published online: 14 December 2018

(c) The Author(s) 2018

\begin{abstract}
Congenital hearing impairment has a negative effect on the social and communication skills in the majority of children. We compared the development of children with various levels of hearing loss who were supplied with hearing aids and children with cochlear implants and those with no hearing problems. We verified the hypothesis about the bidirectional dependency relationship between the tested variables: the influence of mothers' anxiety on their hearing impaired children's development and the influence of the children's developmental level on the anxiety level of their mothers. The participants were 94 children aged 12 to 24 months with various levels of hearing loss corrected with hearing aids or cochlear implants as well as their mothers. The level of anxiety in mothers was assessed using the State-Trait Anxiety Inventory (STAI). A cause and effect relationship was shown between the child's hearing loss and their development in the communicative area as well as in the social and emotional area. It was found that children with profound hearing impairment with a cochlear implant functioned better than the group of children with similar or lower hearing impairment using hearing aids. The mothers' anxiety conditioned the child's poorer development, and the child's delayed development had an influence on increasing the anxiety level in mothers. The level of profoundness of hearing loss weakens communicative as well as social and emotional development of children. There exists a bidirectional relationship between mothers' anxiety and their children's development.
\end{abstract}

Keywords Hearing impairment $\cdot$ Stress $\cdot$ Mothers' anxiety $\cdot$ Children's development $\cdot$ Cochlear implant

Efficient hearing allows the child to develop perceptions of acoustic stimuli and thereby to become aware of the surrounding world. The child gets to know the world, amongst other things, through its acoustic features, and they also learn how to use sounds to communicate with other people. It is assumed that the first 2 years are of essential importance for the development (YoshinagaItano 1999) of the communicative process and the actions taken by mothers during this period may significantly inhibit, limit or delay speech development. Impaired communication has a negative influence on global development-both intellectual and social (Cantwell and

Agnieszka Pilarska

apilarska@ump.edu.pl

1 Poznan University of Medical Sciences, Poznan, Poland

2 Clinical Department of Phoniatrics and Audiology, Heliodor Święcicki Clinical Hospital of the Poznan University of Medical Sciences, Poznan, Poland
Baker 1980). The early detection of a hearing defect in a child and the appropriate emotional interactions with the mother along with professional treatment and rehabilitation are very important for the child's development (Pruszewicz and Sekula 1996).

Diagnosis of hearing loss in a child, however, is usually a shock for the mother and it may induce an emotional crisis that unfavourably affects her disabled child (Weisel et al. 2007). Reactions to the information about disability are individual, however, in which the common emotions are, amongst other things, anxiety, despair, regret, anger and aggression. Sometimes the denial mechanism is also activated. The literature emphasises the complexity of the emotional crisis phenomenon (Zaidman-Zait and Most 2005). However, the essence of the crisis, as a disruption of the balance, is characterised by a sense of helplessness, cognitive chaos, unrealistic perception and experience of reality, a sudden increase in tension, disorganisation in psychosocial functioning and absence of effective methods for handling the situation (Caplan 1963). Research shows that becoming aware of the child's hearing loss may be a 
factor which considerably reduces the parents' potential and poses a risk for their mental health. Mothers of younger, 22 month old, deaf children reported higher pessimism about their children's achieving self-sufficiency and concerns about their children's communication abilities than mothers of hearing children. When their children were 3 and 4 years old, mothers of deaf and hearing children did not differ in their reports of general parenting stress, as measured by the Parenting Stress Index (PSI) (Lederberg and Golbach 2002).

The following types of maternal attitudes which appear the moment mothers become aware of the fact that they have a disabled child may be distinguished: excessively protective attitude, passive/evasive attitude, unstable attitude, overly demanding attitude and accepting attitude. It is assumed that the accepting attitude is a beneficial attitude which promotes the correct development and effective rehabilitation of a child with hearing loss (Weisel et al. 2007). Research by Pruszewicz and Sekula (1996) has shown that factors which contribute to a failure of rehabilitation include: profound hearing loss, late diagnosis of hearing loss, late onset of rehabilitation of the child, lack of appropriate care on the part of the parents. As a result, the child may not be able to attend a regular school, which has a disadvantageous effect on their correct cognitive and social development as well as on the parents' social perspective (Pruszewicz and Sekula 1996).

Accepting the fact of being a mother of a child with hearing loss is a difficult and long-term process. It requires dealing with many emotions. However, ultimately, it has a significant influence on building relations with the child, emotional closeness, the quality of the emotional bond and the formation of the child's own identity (Sen and Yurtsever 2007). Most mothers with a child with chronic physical illness adapt well with regard to the parent-child relationship and parenting behaviours and styles. Nonetheless, some mothers of children with chronic diseases, including hearing impairment, may have difficulties finding appropriate levels of protective behaviours, control, and parental warmth as well as building positive mutual relationships with their child (Pinquart 2013). Interpersonal factors (attachment) and social-ecological factors (social support) were crucial for maternal adaptation. Both constitute 'risk resistant' factors which are mediated by the mother's perceived general stress and guilt (Findler et al. 2016). Mothers who, at a given time, are incapable of accepting their child's disability very often cannot accompany the child in their development and do not notice the child's strengths, which realistically translates into the process of implementing hearing and speech rehabilitation (Pruszewicz and Sekula 1996). In addition, mothers with additionally handicapped children are especially stressed and the child's communicative competence makes for a more sound prediction than its linguistic medium (spoken language or sign) (Hintermair 2006).

The use of an appropriate emotional and rehabilitation path is the turning point for the quality of life and an opportunity for the normal development of deaf child, which also influences the mothers of a child with hearing loss: it reduces their anxiety and stress levels, motivates them to undertake rehabilitation and gives them the strength to overcome difficulties which may appear in the process (Dolgin et al. 1990). This study was conducted to compare the development of children with various levels of hearing loss and rehabilitation paths, i.e. supplied with hearing aids and children with cochlear implants. We verified the hypothesis about the bidirectional emotional dependency manifested by the influence of mothers' anxiety on their hearing impaired children's development and the influence of the children's developmental level on the anxiety level of their mothers.

\section{Method}

\section{Participants}

Ninety-four children at various levels of hearing screening took part in the study. The study included children at ages ranging from 12 to 24 months together with their mothers $(\mathrm{AGE}=18.85 \pm 4.24$ months) (Table 1$)$. In the analysed group, 43 children had profound hearing loss, including 21 with cochlear implants $(\mathrm{CI})\left(\mathrm{AGE}_{\mathrm{CI}}=19.52 \pm 4.28\right.$ months; $\min =13$ months; $\max =24$ months $)$ and 23 with hearing aids (HA) $\quad\left(\mathrm{AGE}_{\mathrm{HA}}=19.09 \pm 4.90\right.$ months; $\min =$ 12 months; $\max =24$ months); 23 children had mild to moderate hearing loss $(\mathrm{MH}) \quad\left(\mathrm{AGE}_{\mathrm{MH}}=17.70 \pm\right.$

Table 1 Descriptive statistics of age and developmental quotients in all children participated in the study with different hearing damages and enhancing devices

\begin{tabular}{lrrrrrr}
\hline & AGE & GMS & \multicolumn{1}{l}{ FMS } & \multicolumn{1}{c}{ SED } & COM & \multicolumn{1}{c}{ DEV } \\
\hline$N$ & \multicolumn{1}{c}{94} & \multicolumn{1}{l}{94} & \multicolumn{1}{l}{94} & \multicolumn{1}{l}{94} & \multicolumn{1}{l}{94} & \multicolumn{1}{l}{94} \\
Mean & 18.85 & 94.41 & 92.94 & 86.16 & 74.13 & 86.69 \\
Std. Dev. & 4.24 & 14.11 & 12.75 & 16.86 & 21.60 & 15.06 \\
Median & 19.00 & 93.50 & 91.50 & 89.00 & 78.00 & 90.00 \\
Minimum & 12.00 & 68.00 & 60.00 & 25.00 & 23.00 & 48.00 \\
Maximum & 24.00 & 125.00 & 120.00 & 125.00 & 125.00 & 120.00 \\
Mode & 24.00 & 100.00 & 90.00 & 100.00 & 70.00 & 90.00 \\
Skewness & -0.20 & 0.17 & 0.02 & -0.83 & -0.17 & -0.36 \\
Kurtosis & -1.41 & -0.59 & -0.69 & 1.83 & -0.32 & -0.42 \\
\hline Developm & & & & & &
\end{tabular}

Developmental quotients: $A G E$ age in months, GMS Gross Motor Skills, FMS Fine Motor Skills, SED Social and Emotional Development, COM Communication, DEV Global Developmental Quotient 
Table 2 Developmental quotients of children with different hearing damages and enhancing devices assessed by the INSITE Developmental Scale

\begin{tabular}{lllllllll}
\hline HearingDamage & $\mathrm{N}$ & \multicolumn{1}{l}{$\%$} & \multicolumn{1}{l}{ AGE } & \multicolumn{1}{l}{ GMS } & \multicolumn{1}{l}{ FMS } & \multicolumn{1}{l}{ SED } & COM & DEV \\
\hline CI & 21 & 22.3 & $19.52 \pm 4.28 \mathrm{a}$ & $100.42 \pm 7.86 \mathrm{a}$ & $100.24 \pm 10.78 \mathrm{a}$ & $88.95 \pm 11.54 \mathrm{a}$ & $72.62 \pm 7.12 \mathrm{a}$ & $89.57 \pm 8.28 \mathrm{a}$ \\
$\mathrm{HA}$ & 23 & 24.5 & $19.09 \pm 4.90 \mathrm{a}$ & $81.78 \pm 8.21 \mathrm{~b}$ & $82.39 \pm 7.83 \mathrm{~b}$ & $75.00 \pm 17.15 \mathrm{~b}$ & $62.09 \pm 24.46 \mathrm{ac}$ & $75.09 \pm 13.50 \mathrm{~b}$ \\
MH & 23 & 24.5 & $17.70 \pm 3.59 \mathrm{a}$ & $88.10 \pm 5.44 \mathrm{~b}$ & $87.57 \pm 4.78 \mathrm{~b}$ & $84.87 \pm 8.19 \mathrm{ac}$ & $80.70 \pm 9.62 \mathrm{ab}$ & $85.65 \pm 5.24 \mathrm{ac}$ \\
EH & 27 & 28.7 & $19.11 \pm 4.16 \mathrm{a}$ & $100.60 \pm 2.50 \mathrm{a}$ & $97.19 \pm 9.48 \mathrm{a}$ & $96.30 \pm 10.87 \mathrm{ad}$ & $90.85 \pm 12.77 \mathrm{~b}$ & $96.70 \pm 9.16 \mathrm{ad}$ \\
Total & 94 & 100 & $18.86 \pm 4.24$ & $92.89 \pm 12.10$ & $91.89 \pm 10.99$ & $86.65 \pm 14.48$ & $77.26 \pm 18.39$ & $87.12 \pm 12.33$ \\
\hline
\end{tabular}

Developmental quotients: AGE age in months, GMS Gross Motor Skills, FMS Fine Motor Skills, SED Social and Emotional Development, COM Communication, DEV Global Developmental Quotient, Hearing damages: CI Profound Hearing Loss with Cochlear Implant, HA Profound Hearing Loss with Hearing Aids, $M H$ Mild to Moderate Hearing Loss without Enhancing Devices, $E H$ Efficient Hearing

Different letters indicate statistically significant differences $(p<0.05)$

3.59 months; $\min =13$ months; $\max =24$ months) and 27 children had efficient hearing $(\mathrm{EH})\left(\mathrm{AGE}_{\mathrm{EH}}=19.11 \pm\right.$ 4.16 months; $\min =13$ months; $\max =24$ months $]$ despite suspected hearing loss in screening tests (Table 2). The diagnosis of hearing loss and the HA rehabilitation was done in 3-6 months. CI were implanted between 12-18 months of age.

\section{Procedure}

The data were collected in 2013-2015 at the Clinical Department of Phoniatrics and Audiology, Heliodor Święcicki Clinical Hospital of the Poznan University of Medical Sciences in Poland. Mothers and children were asked to fill out a questionnaire online or a printed version. The hearing loss level in the children was determined on the basis of objective hearing tests conducted by an audiologist within hearing screening stages.

\section{Measures}

The research methodology included an assessment of the child's developmental level using the INSITE Developmental Scale and next the mother filled in the following questionnaires: State-Trait Anxiety Inventory (STAI).

\section{Level of the child's development}

The child's level of developmental skills was assessed using the INSITE Developmental Scale-a developmental scale for infants and small children with sensory disability and additional defects aged 0-6 years of age (Morgan et al. 1989). The scale is based on the development of healthy children, but it is adapted to the requirements of children with sensory disability and additional defects according to their specific developmental functioning. To compare the level of development of the tested persons, the following method of calculating the developmental quotient for a given child was used:
Developmental quotient $(\mathrm{DQ})=$ Child's developmental age (DA)/Child's age (CA) x 100

The developmental age and the child's age is provided in days. For individual developmental areas, the partial DQ was determined, and the DQ for the child's global development was determined by taking into account the partial DQ and, on their basis, the averaged level of the child's functioning was established.

\section{Anxiety levels in mothers}

The State-Trait Anxiety Inventory (STAI) (Spielberger et al. 1970) as adapted into Polish by Sosnowski and Wrześniewski (Sosnowski and Wrześniewski 1983) was used to examine the anxiety level. The research tool consists of two subscales in which one $(\mathrm{X}-1)$ is used to measure the anxiety-state and the other (X-2) anxiety-trait. Each subscale consists of 20 items to which the subject responds choosing one of four categorised responses (1-definitely no, 2-rather no, 3-rather yes, 4-definitely yes). The study measured the level of anxiety as a state, so only subscale X-1 was used. The anxiety level was expressed by the number of points obtained by summing up the scores for individual responses. The point values may fall within the range of 20 to 80 . The growing point values indicate elevated anxiety levels.

\section{Child's hearing loss}

The child's hearing level was classified as a nominal variable in the following way: Efficient hearing-hearing threshold $<40 \mathrm{~dB}$ at least in one ear $(\mathrm{EH})$; Mild to moderate hearing loss (loss $\geq 40<80 \mathrm{~dB}$ ) ( $\mathrm{MH})$; Profound hearing loss (loss $\geq 80 \mathrm{~dB}$ ) treating with hearing aids (HA); Profound hearing loss (loss $\geq 80 \mathrm{~dB}$ ) with a cochlear implant (CI). For the aforementioned groups, the profoundness of hearing loss was determined according to the hearing level of the better ear for asymmetrical hearing loss. 


\section{Data analysis}

The statistical analysis was conducted using raw results for dependent variables on a continuous scale, and zero hypotheses on no differences were tested with a parametric one-way analysis of variance (ANOVA). The assumption about normal distributions in the compared groups was verified using the Lilliefors-corrected KolmogorovSmirnov test and the Shapiro-Wilk test, and the homogeneity of variance using the Brown-Forsythe test. In situations in which significant variance differences were found, post-hoc comparisons were made using Tukey's significant difference test modified by Spjotvoll and Stoline for groups of different sizes. The statistical significance in all of the aforementioned tests was adopted at $p<0.05$. All statistical calculations were performed with Statistica 6.0 software (StatSoft).

\section{Results}

\section{Hearing Loss and the Child's Gross Motor Skills (GMS)}

The ANOVA analysis of variance showed significant differences at the significance level of $p<0.05$ between the considered means of the global developmental quotient variable in the individual groups of the hearing loss in the child independent variable. In post-hoc inter-group comparisons between individual groups, significant differences were found in the gross motor skills variable between the following groups of hearing loss in the child independent variable: $\mathrm{CI}$ vs. $\mathrm{HA}\left(\mathrm{GMS}_{\mathrm{CI}}=100.42 \pm 7.86 ; \mathrm{GMS}_{\mathrm{HA}}=\right.$ $81.78 \pm 8.21), \quad \mathrm{CI} \quad$ vs. $\mathrm{MH} \quad\left(\mathrm{GMS}_{\mathrm{CI}}=100.42 \pm 7.86\right.$; $\left.\mathrm{GMS}_{\mathrm{MH}}=88.10 \pm 5.44\right)$, HA vs. $\mathrm{EH}\left(\mathrm{GMS}_{\mathrm{HA}}=81.78 \pm\right.$ $\left.8.21 ; \mathrm{GMS}_{\mathrm{EH}}=100.60 \pm 12.50\right)$ and $\mathrm{MH}$ vs. $\mathrm{EH}\left(\mathrm{GMS}_{\mathrm{MH}}\right.$ $\left.=88.10 \pm 5.44 ; \mathrm{GMS}_{\mathrm{EH}}=100.60 \pm 12.50\right)$. Children with efficient hearing $(\mathrm{EH})$ and profound hearing loss with a cochlear implant (CI) belonged to groups presenting the best results in the considered variable. The poorest group in terms of global development were children with profound hearing loss with hearing aids (HA) (Table 2).

\section{Hearing Loss and the Child's Fine Motor Skills (FMS)}

The analysis of variance (ANOVA) showed significant differences at $p<0.05$ between the considered means of the fine motor skills variable in the individual groups of the hearing loss level in the child independent variable. On the basis of inter-group post-hoc comparisons, significant differences for the tested variable were found between the following groups of variables $\mathrm{CI}$ vs. $\mathrm{HA} \quad\left(\mathrm{FMS}_{\mathrm{CI}}=\right.$ $\left.100.24 \pm 10.78 ; \quad \mathrm{FMS}_{\mathrm{HA}}=82.39 \pm 7.83\right), \quad \mathrm{CI}$ vs. $\mathrm{MH}$
$\left(\mathrm{FMS}_{\mathrm{CI}}=100.24 \pm 10.78 ; \mathrm{FMS}_{\mathrm{MH}}=87.57 \pm 4.78\right), \mathrm{HA}$ vs. $\mathrm{EH}\left(\mathrm{FMS}_{\mathrm{HA}}=82.39 \pm 7.83 ; \mathrm{FMS}_{\mathrm{EH}}=97.19 \pm 9.48\right)$ and $\mathrm{MH}$ vs. $\mathrm{EH}\left(\mathrm{FMS}_{\mathrm{MH}}=87.57 \pm 4.78 ; \mathrm{FMS}_{\mathrm{EH}}=97.19 \pm\right.$ 9.48). The results of the analysis showed that children with efficient hearing $(\mathrm{EH})$ and children with a cochlear implant (CI) developed the best among the analysed groups (Table 2).

\section{Hearing Loss and the Child's Social and Emotional Development (SED)}

The conducted ANOVA analysis of variance showed significant differences at $p<0.05$ between the considered means of the social and emotional development variable in the individual groups of the hearing loss level in the child independent variable. On the basis of inter-group post-hoc comparisons, significant differences for the tested variable were found between the following groups of variables CI vs. HA $\left(\mathrm{SED}_{\mathrm{CI}}=88.95 \pm 11.54 ; \mathrm{SED}_{\mathrm{HA}}=75.00 \pm 17.15\right)$, $\mathrm{HA}$ vs. $\mathrm{MH}\left(\mathrm{SED}_{\mathrm{HA}}=75.00 \pm 17.15 ; \mathrm{SED}_{\mathrm{MH}}=84.87 \pm\right.$ $8.19), \mathrm{HA}$ vs. $\mathrm{EH}\left(\mathrm{SED}_{\mathrm{HA}}=75.00 \pm 17.15 ; \mathrm{SED}_{\mathrm{EH}}=\right.$ $96.30 \pm 10.87)$ and $\mathrm{MH}$ vs. $\mathrm{EH}\left(\mathrm{SED}_{\mathrm{MH}}=84.87 \pm 8.19\right.$; $\left.\mathrm{SED}_{\mathrm{EH}}=96.30 \pm 10.87\right)$. The results of the analysis showed that children with efficient hearing $(\mathrm{EH})$ developed the best among the analysed groups. It was also found that children with profound hearing loss achieved poorer results of development after receiving a cochlear implant (CI) than the group of children with efficient hearing (EH). Group HA with profound hearing loss was developed the poorest in terms of the tested variable. The research results confirm observations and assumptions that a greater hearing loss in small children causes poorer social and emotional development, amongst other things, due to the limited ability to understand messages from their surroundings and, as a result, to read and respond correctly to social situations. Children with a cochlear implant (CI) achieved higher results on a developmental scale as regards the tested variable in the group of children with profound hearing loss with hearing aids (Table 2).

\section{Hearing Loss on the Child's Communicative Development (COM)}

The ANOVA analysis of variance showed significant differences at $p<0.05$ between the considered means of the communication development variable in the individual groups of the Hearing loss level in the child independent variable. This seems obvious as hearing loss has an inhibitory effect on the development of communicative processes. In post-hoc comparisons between individual groups, significant differences were found in the communication variable between the following groups of the Hearing loss level in the child independent variable: CI vs. EH 
$\left(\mathrm{COM}_{\mathrm{CI}}=72.62 \pm 7.12 ; \mathrm{COM}_{\mathrm{EH}}=90.85 \pm 12.77\right), \mathrm{HA}$ vs. $\mathrm{MH} \quad\left(\mathrm{COM}_{\mathrm{HA}}=62.09 \pm 24.46 ; \quad \mathrm{COM}_{\mathrm{MH}}=80.70 \pm 9.62\right)$ and $\mathrm{HA}$ vs. $\mathrm{EH}\left(\mathrm{COM}_{\mathrm{HA}}=62.09 \pm 24.46 ; \mathrm{COM}_{\mathrm{EH}}=\right.$ $90.85 \pm 12.77)$. The best developing group in terms of the communicativeness level were children with efficient hearing (EH). Children with profound hearing loss with hearing aids (HA) and children with cochlear implants (CI) developed worse in terms of the achieved level of the communicative process (Table 2).

\section{Hearing Loss and the Child's Global Development (DEV)}

The ANOVA analysis of variance showed significant differences at $p<0.05$ between the considered means of the global development variable in the individual groups of the Hearing loss level in the child independent variable. In posthoc comparisons between individual groups, significant differences were found in the global development variable between the following groups of the hearing loss level in the child independent variable: $\mathrm{CI}$ vs. $\mathrm{HA}\left(\mathrm{DEV}_{\mathrm{CI}}=89.57 \pm\right.$ 8.28; $\left.\mathrm{DEV}_{\mathrm{HA}}=75.09 \pm 13.50\right)$, HA vs. $\mathrm{MH}\left(\mathrm{DEV}_{\mathrm{HA}}=\right.$ $\left.75.09 \pm 13.50 ; \mathrm{DEV}_{\mathrm{MH}}=85.65 \pm 5.24\right)$ and $\mathrm{HA}$ vs. $\mathrm{EH}$ $\left(\mathrm{DEV}_{\mathrm{HA}}=75.09 \pm 13.50 ; \quad \mathrm{DEV}_{\mathrm{EH}}=96.70 \pm 9.16\right)$. Children with profound hearing loss with hearing aids (HA) developed worse in terms of the achieved level of the global development process (Table 2).

\section{Hearing Loss and Mothers' Anxiety (ANX)}

The ANOVA analysis of variance showed significant differences at $p<0.05$ between the considered means of the mothers' anxiety variable in the individual groups of the hearing loss level in the child independent variable. In posthoc comparisons between individual groups, significant differences were found in the mothers' anxiety variable between the following groups of the hearing loss level: CI vs. $\mathrm{HA}\left(\mathrm{ANX}_{\mathrm{CI}}=33.33 \pm 5.80 ; \mathrm{ANX}_{\mathrm{HA}}=70.87 \pm 3.77\right)$, $\mathrm{HA}$ vs. $\mathrm{MH}\left(\mathrm{ANX}_{\mathrm{HA}}=70.87 \pm 3.77 ; \mathrm{ANX}_{\mathrm{MH}}=43.48 \pm\right.$ $12.80)$ and $\mathrm{HA}$ vs. $\mathrm{EH}\left(\mathrm{ANX}_{\mathrm{HA}}=70.87 \pm 3.77 ; \mathrm{ANX}_{\mathrm{EH}}=\right.$ $27.67 \pm 7.18$ ). Mothers of children with profound hearing loss with hearing aids (HA) were shown to have the highest anxiety level $\left(\mathrm{ANX}_{\mathrm{HA}}=70.87 \pm 3.77\right)$ (Fig. 1).

\section{Mothers' Anxiety and Global Development (DEV)}

The conducted ANOVA analysis of variance showed significant differences at $p<0.05$ between the considered means of the mothers' anxiety levels variable in the individual groups of the global developmental quotient variable. Post-hoc comparisons have revealed significant differences at $p<0.05$ in the developmental variable between the groups of the anxiety variable: low anxiety vs. medium

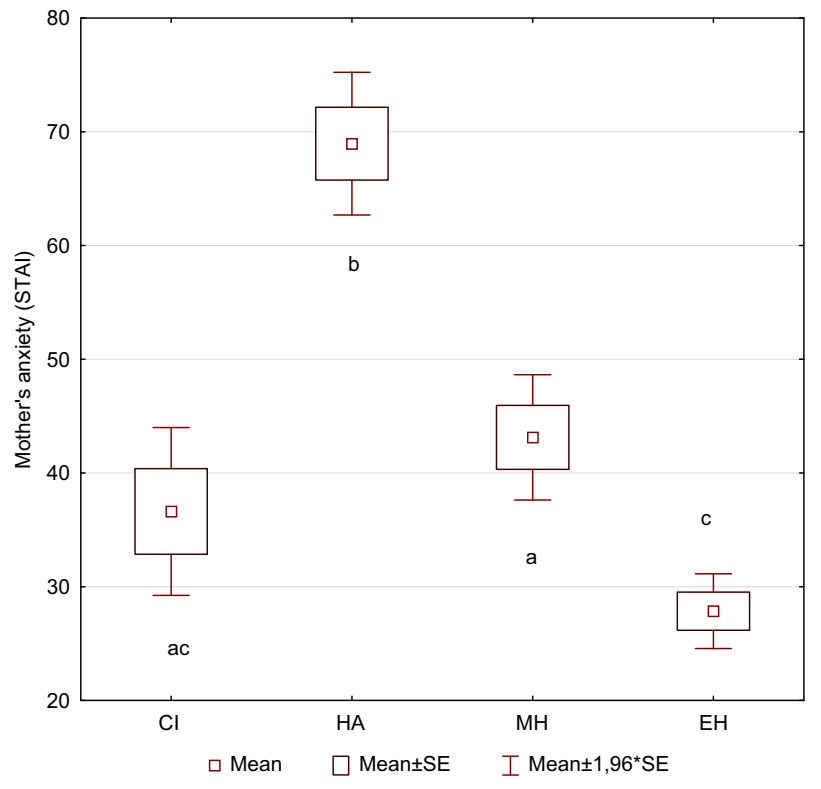

Fig. 1 Mother's anxiety level grouped by children with different hearing damages and enhancing devices. CI-Profound Hearing Loss with Cochlear Implant, HA-Profound Hearing Loss with Hearing Aids, MH-Mild to Moderate Hearing Loss without Enhancing Devices, EH-Efficient Hearing. Different letters indicate statistically significant differences $(p<0.05)$

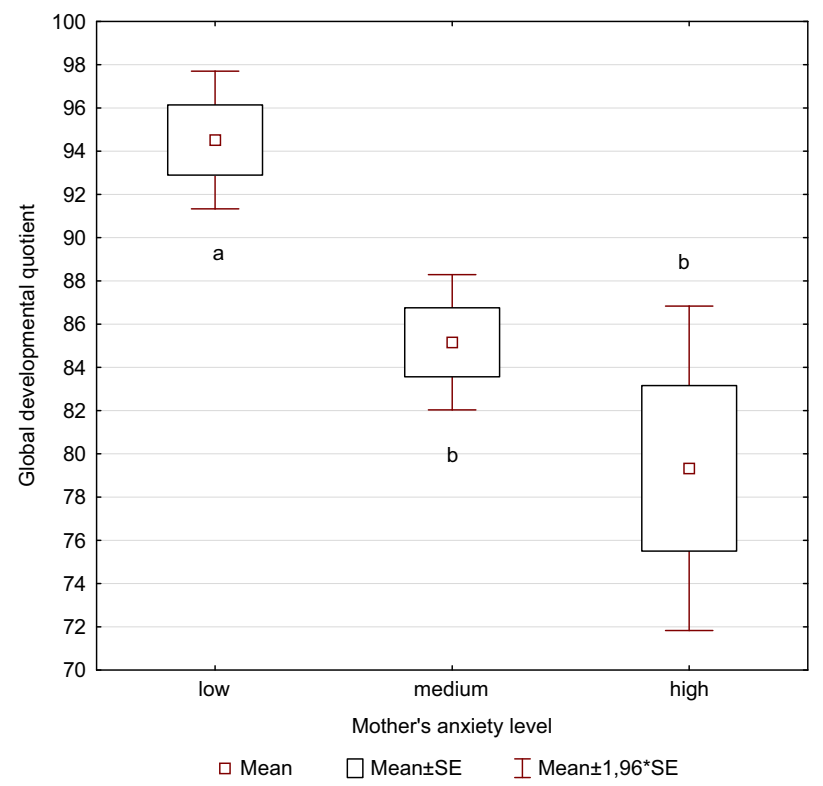

Fig. 2 Global development quotient of children grouped by mother's anxiety level determined by STAI questionnaire. Different letters indicate statistically significant differences $(p<0.05)$

anxiety $\quad\left(\mathrm{DEV}_{\mathrm{LANX}}=94.52 \pm 8.74 ; \quad \mathrm{DEV}_{\mathrm{MANX}}=85.16 \pm\right.$ $11.28)$ and low anxiety vs. high anxiety $\left(\mathrm{DEV}_{\mathrm{LANX}}=\right.$ $\left.94.52 \pm 8.74 ; \mathrm{DEV}_{\mathrm{HANX}}=79.33 \pm 14.82\right)$. No statistically significant differences were observed between the medium anxiety and high anxiety groups $\left(\mathrm{DEV}_{\mathrm{MANX}}=85.16 \pm\right.$ 11.28; $\mathrm{DEV}_{\mathrm{MANX}}=79.33 \pm 14.82$ ) (Fig. 2). 


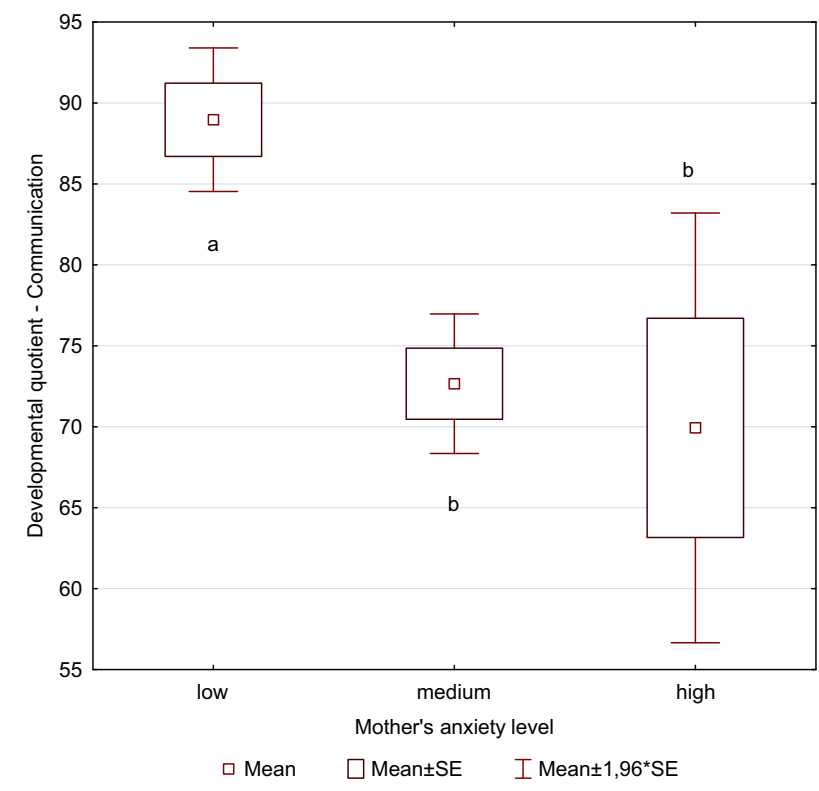

Fig. 3 Communication skills of children grouped by mother's anxiety level determined by STAI questionnaire. Different letters indicate statistically significant differences $(p<0.05)$

\section{Mothers' Anxiety and Communicative Development (COM)}

The ANOVA analysis of variance showed significant differences at $p<0.05$ between the considered means of the anxiety level variable in the individual groups of the communicative development variable (COM). Inter-group post-hoc comparisons have revealed significant differences in the child's communicative development variable between the groups of the mother trait anxiety levels at $p<$ 0.05: low anxiety vs. medium anxiety $\left(\mathrm{COM}_{\mathrm{LANX}}=\right.$ $\left.88.96 \pm 12.18 ; \quad \mathrm{COM}_{\mathrm{MANX}}=72.66 \pm 15.54\right)$ and low anxiety vs. high anxiety $\left(\mathrm{COM}_{\mathrm{LANX}}=88.96 \pm 12.18\right.$; $\mathrm{COM}_{\mathrm{HANX}}=69.93 \pm 26.22$ ). No statistically significant differences were found between the medium anxiety and high anxiety $\left(\mathrm{COM}_{\mathrm{MANX}}=72.66 \pm 15.54 ; \mathrm{COM}_{\mathrm{HANX}}=\right.$ $69.93 \pm 26.22)$ (Fig. 3).

\section{Mothers' Anxiety and Fine Motor Skills (FMS)}

The conducted ANOVA analysis of variance showed significant differences at $p<0.05$ between the considered means of the anxiety level variable in the individual groups of the fine motor skills variable. Post-hoc comparisons have revealed significant $(p<0.05)$ differences in the developmental variable between the groups of the anxiety variable: low anxiety vs. medium anxiety $\left(\mathrm{FMS}_{\mathrm{LANX}}=97.37 \pm 11.64\right.$; FMS $\left._{\text {MANX }}=90.58 \pm 10.19\right)$ and low anxiety vs. high anxiety $\left(\mathrm{FMS}_{\mathrm{LANX}}=97.37 \pm 11.64 ; \mathrm{FMS}_{\mathrm{HANX}}=85.67 \pm 7.58\right)$. No statistically significant differences were found between

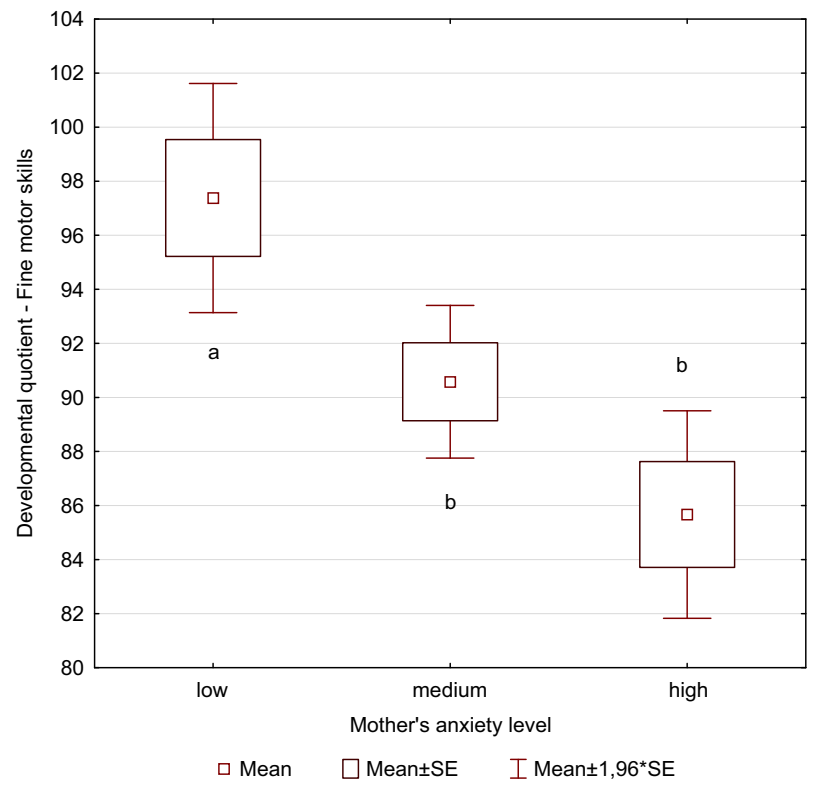

Fig. 4 Fine motor skills of children grouped by mother's anxiety level determined by STAI questionnaire. Different letters indicate statistically significant differences $(p<0.05)$

the medium anxiety and high anxiety $\left(\mathrm{FMS}_{\mathrm{MANX}}=90.58 \pm\right.$ 10.19; $\mathrm{FMS}_{\mathrm{HANX}}=85.67 \pm 7.58$ ) (Fig. 4).

\section{Mothers' Anxiety and Gross Motor Skills (GMS)}

The conducted ANOVA analysis of variance showed significant differences at the significance level $p<0.05$ between the considered means of the anxiety level variable in the individual groups of the gross motor skills independent variable. Inter-group post-hoc comparisons have revealed significant differences in the gross motor skills variable between the groups of the anxiety variable at $p<$ 0.05: low anxiety vs. high anxiety $\left(\mathrm{GMS}_{\mathrm{LANX}}=98.51 \pm\right.$ 11.24; $\mathrm{GMS}_{\mathrm{HANX}}=84.47 \pm 8.56$ ). No statistically significant differences were found between the other groups (Fig. 5).

\section{Mothers' Anxiety and Social and Emotional Development (SED)}

The conducted ANOVA analysis of variance showed significant differences at $p<0.05$ between the considered means of the anxiety level variable in the individual groups of the social and emotional development variable. Post-hoc comparisons have revealed significant $(p<0.05)$ differences in the developmental variable between the groups of the anxiety variable: low anxiety vs. medium anxiety $\left(\operatorname{SED}_{\mathrm{LANX}}=94.90 \pm 11.16 ; \quad \operatorname{SED}_{\mathrm{MANX}}=84.46 \pm 12.22\right)$ and low anxiety vs. high anxiety $\left(\mathrm{SED}_{\mathrm{LANX}}=94.90 \pm\right.$ 11.16; $\quad \mathrm{SED}_{\mathrm{HANX}}=78.00 \pm 19.56$ ). No statistically 


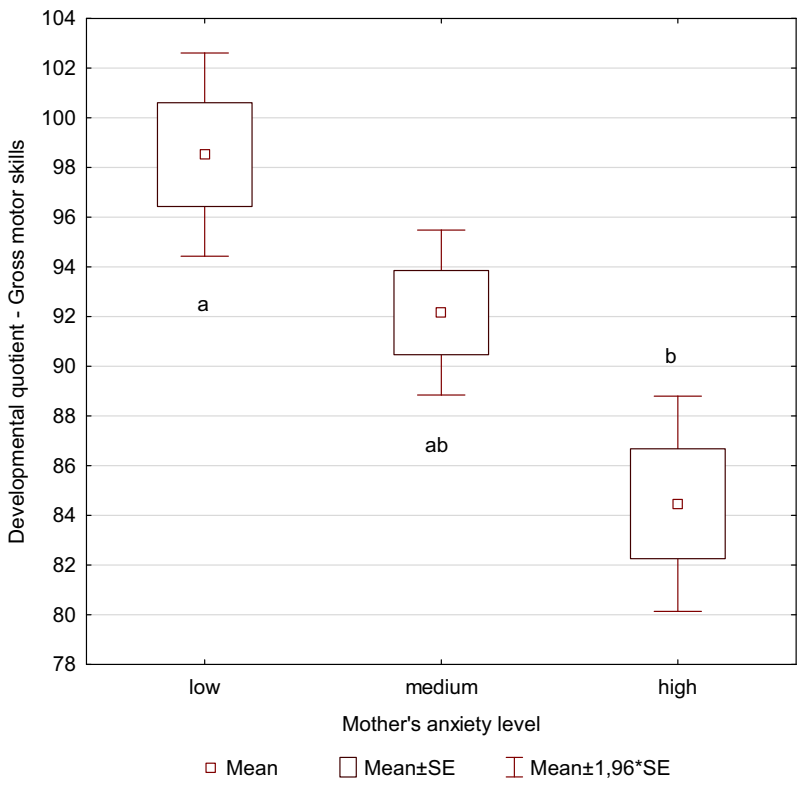

Fig. 5 Gross motor skills of children grouped by mother's anxiety level determined by STAI questionnaire. Different letters indicate statistically significant differences $(p<0.05)$

significant differences were found between medium anxiety and high anxiety $\left(\mathrm{SED}_{\mathrm{MANX}}=84.46 \pm 12.22 ; \mathrm{SED}_{\mathrm{HANX}}=\right.$ $78.00 \pm 19.56$ ) (Fig. 6).

\section{Discussion}

In this study, an analysis of the influence of hearing loss on the individual developmental areas of a child was presented together with a bidirectional dependency relationship between mothers' anxiety and the development of their children with hearing loss. The study was conducted in mothers and their children between 1 and 2 years of age. An influence of hearing loss in children on their development in individual developmental areas was demonstrated, especially in communication and social and emotional development. The child's poorer development was related to a more profound hearing loss. However, the use of a cochlear implant-by maximum elimination of the effects of profound hearing loss-improved the child's functioning in the analysed areas. Therefore, it can be concluded that the profoundness of hearing loss was a significant component of a broader aspect of the child's disability which caused a specific reception of this situation by the children's mothers.

Some data confirm that a disability diagnosis-including a child's hearing loss-is a shock for parents and it is accompanied by a lot of strong and negative emotions resulting from the experience of loss (Hoff 1995). This may lead to a severe disruption of the mental balance, including a sudden increase in the parents' anxiety (Caplan 1963;

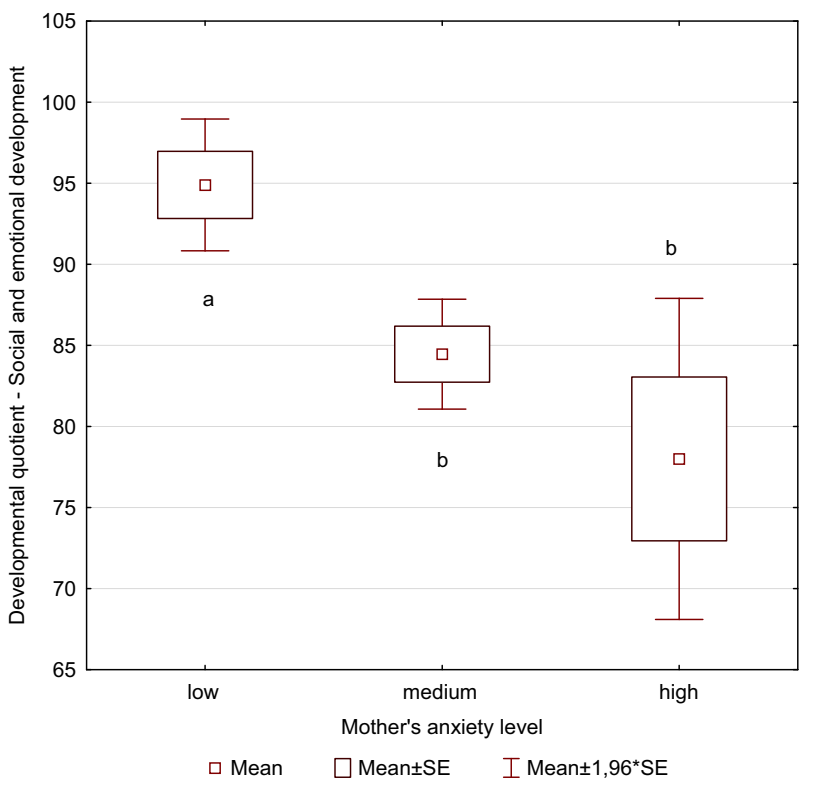

Fig. 6 Social and emotional development of children grouped by mother's anxiety level determined by STAI questionnaire. Different letters indicate statistically significant differences $(p<0.05)$

Lindemann 1944). Research conducted by Pruszewicz and Sekula (Pruszewicz and Sekula 1996) revealed that parents who presented various attitudes in this situation-from denial to overprotective-achieved different results in the rehabilitation of the communication process in their children.

The results presented in the present paper emphasise that the mothers' mental state is directly related to the psychophysical status of their children. It seems that the influence of children's auditory disability on their mothers' emotions is related to the chronic nature of the experienced anxiety and stress. Under prolonged tension, mothers may begin to function more poorly after some time also on other planes, which is caused by higher susceptibility to stress. High maternal stress is associated with frequent socioemotional problems in the children, thus emphasising the importance of a resource-oriented consulting and support strategy in early intervention because parental access to personal and social resources is associated with significantly lower stress (Hintermair 2006).

Many researchers have confirmed that a prolonged period of an excessive number of duties, excessive tiredness and lack of support causes interpersonal conflicts and an increase in anxiety and stress (Dohrenwend and Dohrenwend 1982; Plopa and Makarowski 2010). Plopa and Makarowski add that, if the mental system is filled with conflicts and tension, no external stressors are required to feel threatened after some time. This is related to lower levels of an individual's resources, which are understood at broad biological properties (e.g. the function of the immune 
system) or psychological properties (cognitive, intellectual, personality functions, coping with stress, the sense of identity) (Plopa and Makarowski 2010).

The literature on this subject shows that a child's hearing loss can be a factor which poses a risk for the mothers' mental health (Kobosko et al. 2014). Compared to mothers of correctly developing children, mothers of children with impaired hearing may exhibit more depressive symptoms, including elevated anxiety levels (Kobosko et al. 2014; Quittner et al. 1991). The relationship between the needs of mothers who have hearing impairment children and their state-trait anxiety level was reviewed by Bilsin et al. (2015). They identified the relationship between the needs of those mothers who have hearing impairment children and their state/trait anxiety levels. Significant positive relationships were found between the mothers' state anxiety level and the overall FNS score, the subscales of need for information, help explaining to others, community services, financial assistance and family functioning as well as a significant positive relationship were found between the anxiety level and the overall FNS score, the subscales of need for information, need for support, help explaining to others community services, financial assistance and family functioning (Bilsin et al. 2015). Furthermore, mothers with disabled children felt severe sadness, and they indicated that, after having a disabled child, their social life, working life and family relationships were all affected (Sen and Yurtsever 2007).

Such observations correspond to the results obtained in this study where a relationship was found between hearing loss in children and the level of their development and the anxiety felt by their mothers. The lowest level of anxietycomparable to that in the group of mothers of children with efficient hearing - was felt by mothers with children after the cochlear implant surgery. This means that the cochlear implantation reduced the anxiety indicators. This can be explained by the fact that mothers of children with a cochlear implant stayed at the hospital at least several times, they already knew the examination procedures and they received information and support from physicians and personnel at a suitable level. As shown by clinical experience, the cochlear implant surgery is an important source of anxiety in mothers. At the first stage, the parents face the dilemma of choosing the cochlear implant or using hearing aids (Feher-Prout 1996).

According to many authors (Archbold et al. 2006; Hyde et al. 2010; Weisel et al. 2007), the mothers' expectations related to the cochlear implant are high and they concern a significant improvement in the functioning in the communication area, which may initially influence their improved mood and hopes. This is confirmed by the results obtained by researchers (Anagnostou et al. 2007; Weisel et al. 2007) who describe the intensification of anxiety in mothers before the cochlear implant surgery and a decrease in this intensity after the surgery. At the same time, it is considered that a high level of anxiety is related to a lack of the expected development of their children in regards to communication in a short period of time after the surgery (Allegretti 2002). It was also confirmed that, although mothers of children after implantation show high expectations in the area of communication, social and education development, they are aware of the demanding process of hearing and speech rehabilitation (Zaidman-Zait and Most 2005). Similar results were obtained by Noohi et al. (Noohi et al. 2010), who, in a cohort study, analysed anxiety and depression in mothers of deaf children after receiving a cochlear implant. They found that the level of depression and anxiety was much higher in mothers of children during the process of qualification for the implant compared to mothers of children after the implant surgery.

The cochlear implant surgery led to reduced anxiety levels in mothers. However, they were still higher than in the control group of mothers of children with normal hearing. Similar conclusions were formulated by Gurbuz et al. (2013) on the basis of comparisons of the anxiety levels in parents before and after the cochlear implant surgery. These analyses, regardless of the influence of the cochlear implant surgery on parents' stress, were aimed at defining the importance of personality and sociodemographic factors. These authors found a higher level of anxiety and neuroticism in mothers compared to fathers, which was explained by higher involvement in the care of the child on the part of mothers. At the same time, they showed that the anxiety level falls significantly in both parents after the cochlear implant surgery.

The early detection of a hearing impairment in the child and appropriate intervention in the form of treatment and rehabilitation has a good influence on improving the child's developmental indices, on the one hand, and, on the other hand, on the related emotional functioning of their mothers in the form of reducing the anxiety level, which has a positive influence on the child's development. Particular attention should be paid to the importance of the possibility of using a cochlear implant for children with profound hearing loss, which at present offers the best possibility for children with hearing impairment to master speech via the auditory route. The use of cochlear implants by children with profound hearing loss has an advantageous effect on the child's global development and on the reduction of anxiety levels in their mothers, which, secondarily, contributes to the better stimulation of the child's development.

Author Contributions A.P. designed and executed the study, analysed the data, and wrote the paper. A.S. collaborated with the design and writing of the study. 


\section{Compliance with Ethical Standards}

Conflict of Interest The authors declare that they have no conflict of interest.

Ethical Approval All procedures performed involving human participants were in accordance with the ethical standards of the institutional and/or national research committee and with APA ethical standards.

Informed Consent Informed consent was obtained from all individual participants included in the study (parents).

Open Access This article is distributed under the terms of the Creative Commons Attribution 4.0 International License (http://crea tivecommons.org/licenses/by/4.0/), which permits use, duplication, adaptation, distribution, and reproduction in any medium or format, as long as you give appropriate credit to the original author(s) and the source, provide a link to the Creative Commons license, and indicate if changes were made.

\section{References}

Allegretti, C. M. (2002). The effects of a cochlear implant on the family of a hearing-impaired child. Pediatric Nursing, 28(6), 614-620.

Anagnostou, F., Graham, J., \& Crocker, S. (2007). A preliminary study looking at parental emotions following cochlear implantation. Cochlear Implants International, 8(2), 68-86.

Archbold, S., Sach, T., Lutman, M., \& Gregory, S., et al. (2006). Deciding to have a cochlear implant and subsequent after-care: Parental perspectives. Deafness \& Education International, 8(4), 190-206.

Bilsin, E., Çuhadar, D., \& Göv, P. (2015). A review of the relationship between the needs of mothers who have hearing impairment children and their state-trait anxiety levels. Journal of Pediatric Nursing, 30(1), 254-261.

Cantwell, D. P., \& Baker, L. (1980). Psychiatric and behavioral characteristics of children with communication disorders. Journal of Pediatric Psychology, 5(2), 161-178. https://doi.org/10.1093/ jpepsy/5.2.161.

Caplan, G. (1963). Emotional Crisis. In H. Fishbein \& M. Deutsch (Eds.), The Encyclopedia of Mental Health. New York: Basic Books.

Dohrenwend, B. P., \& Dohrenwend, B. S. (1982). Perspectives on the past and future of psychiatric epidemiology. The 1981 Rema Lapouse Lecture. American Journal of Public Health, 72(11), 1271-1279.

Dolgin, M. J., Phipps, S., Harow, E., \& Zeltzer, L. K. (1990). Parental Management of Fear in Chronically Ill and Healthy Children. Journal of Pediatric Psychology, 15(6), 733-744. https://doi.org/ 10.1093/jpepsy/15.6.733.

Feher-Prout, T. (1996). Stress and coping in families with deaf children. Journal of Deaf Studies and Deaf Education, 1(3), $155-166$.

Findler, L., Klein Jacoby, A., \& Gabis, L. (2016). Subjective happiness among mothers of children with disabilities: The role of stress, attachment, guilt and social support. Research in Developmental Disabilities, 55, 44-54.

Gurbuz, M. K., Kaya, E., Incesulu, A., Gulec, G., Cakli, H., Ozudogru, E., \& Colak, E. (2013). Parental anxiety and influential factors in the family with hearing impaired children: Before and after cochlear implantation. Journal of International Advanced Otology, 9(1), 46-54.

Hintermair, M. (2006). Parental resources, parental stress, and socioemotional development of deaf and hard of hearing children. Journal of Deaf Studies and Deaf Education, 11(4), 493-513.

Hoff, L. A. (1995). People in Crisis: Understanding and Helping. San Francisco: Jossey-Bass.

Hyde, M., Punch, R., \& Komesaroff, L. (2010). Coming to a decision about cochlear implantation: parents making choices for their deaf children. Journal of Deaf Studies and Deaf Education, 15(2), $162-178$.

Kobosko, J., Geremek-Samsonowicz, A., \& Skarżyński, H. (2014). Mental health problems of mothers and fathers of the deaf children with cochlear implants. Otolaryngologia Polska, 68(3), $135-142$.

Lederberg, A., \& Golbach, T. (2002). Parenting stress and social support in hearing mothers of deaf and hearing children: a longitudinal study. Journal of Deaf Studies and Deaf Education, 7(4), 330-345.

Lindemann, E. (1944). Symptomatology and management of acute grief. American Journal of Psychiatry, 101(2), 141-148.

Morgan, E., Watkins, S., \& Terry, B. (1989). Assesment of Development Skills for Young Multihandicapped Sensory Impaired Children. Logan: SKI HI Institute Department of Communication Disorders Utah State University.

Noohi, S., Radfar, S., Tavallaii, S. A., Yousefi, J., Alifard, M. H., \& Ajallouyean, M. (2010). Impact of implantation on anxiety and depression in mothers of children under cochlear implant. Journal of Family and Reproductive Health, 4(4), 187-190.

Pinquart, M. (2013). Do the Parent-Child Relationship and Parenting Behaviors Differ Between Families With a Child With and Without Chronic Illness? A Meta-Analysis. Journal of Pediatric Psychology, 38(7), 708-721.

Plopa, M., \& Makarowski, R. (2010). Kwestionariusz Poczucia Stresu. Warszawa: Wyższa Szkoła Finansów i Zarządzania.

Pruszewicz, A., \& Sekula, A. (1996). Hard-of-hearing children: factors negatively influencing rehabilitation. Folia Phoniatrica et Logopaedica, 48(3), 122-126.

Quittner, A. L., Steck, J. T., \& Rouiller, R. L. (1991). Cochlear implants in children: a study of parental stress and adjustment. Otology \& Neurotology, 12, 95-104.

Sen, E., \& Yurtsever, S. (2007). Difficulties experienced by families with disabled children. Journal for Specialists in Pediatric Nursing, 12(4), 238-252.

Sosnowski, T., \& Wrześniewski, K. (1983). Polska adaptacja inwentarza STAI do badania stanu i cechy lęku. Przeglad Psychologiczny, 26, 393-412.

Spielberger, C. D., Gorsuch, R. L., \& Lushene, R. E. (1970). The State-Trait Anxiety Inventory (test manual). Palo Alto, CA: Consulting Psychologists Press.

Weisel, A., Most, T., \& Michael, R. (2007). Mothers' stress and expectations as a function of time since child's cochlear implantation. Journal of Deaf Studies and Deaf Education, 12(1), $55-64$.

Yoshinaga-Itano, C. (1999). Benefits of early intervention for children with hearing loss. Otolaryngologic Clinics of North America, 32 (6), 1089-1102.

Zaidman-Zait, A., \& Most, T. (2005). Cochlear implants in children with hearing loss: maternal expectations and impact on the family. The Volta Review, 105(2), 129-150. 